\title{
Dietary patterns and blood pressure among middle-aged and elderly Chinese men in Shanghai
}

\author{
Sang-Ah Lee ${ }^{1,2}$, Hui Cai ${ }^{1}$, Gong Yang ${ }^{1}$, Wang-Hong $\mathrm{Xu}^{3}$, Wei Zheng ${ }^{1}$, Honglan $\mathrm{Li}^{3}$, Yu-Tang Gao ${ }^{3}$, \\ Yong-Bing Xiang ${ }^{3}$ and Xiao Ou Shu ${ }^{1 *}$ \\ ${ }^{1}$ Department of Medicine, Vanderbilt Epidemiology Center, Vanderbilt University Medical Center and Vanderbilt-Ingram \\ Cancer Center, 2525 West End Avenue, Suite 600, IMPH, Nashville TN 37203-1738, USA \\ ${ }^{2}$ Department of Preventive Medicine, Kangwon National University, Hyuja2-dong Chucheon-si, Kangwon-do, 110-799 \\ Gangwon-do, South Korea \\ ${ }^{3}$ Department of Epidemiology, Shanghai Cancer Institute, and Cancer Institute of Shanghai Jiao Tong University, No. 25, \\ 2200 Xie Tue Road, Shanghai, PR China
}

(Received 15 April 2009 - Revised 19 January 2010 - Accepted 20 January 2010 - First published online 1 March 2010)

The prevalence of hypertension has increased over the past decade in many developed and developing countries, including China. This increase may be associated with changes in lifestyle, including dietary patterns. We evaluated the association of dietary patterns with blood pressure (BP) by using data from a large, population-based cohort study of middle-aged and elderly Chinese men, the Shanghai Men's Health Study. The present cross-sectional analysis includes 39252 men who reported no prior history of hypertension, diabetes, CHD, or stroke nor use of antihypertensive drugs at study enrolment. Three dietary patterns, 'vegetable', 'fruit and milk' and 'meat', were derived using factor analysis. The fruit and milk diet was inversely associated with both systolic and diastolic BP $\left(P_{\text {trend }}<0 \cdot 001\right)$. The adjusted mean systolic BP was $2.9 \mathrm{mmHg}$ lower $(95 \% \mathrm{CI}-3.4,-2.4)$, and diastolic BP was $1.7 \mathrm{mmHg}$ lower $(95 \% \mathrm{CI}-2.0$, -1.4) for men in the highest quintile of the 'fruit and milk' pattern compared with men in the lowest quintile. This inverse association was more evident among heavy drinkers; the highest quintile of the 'fruit and milk' pattern was associated with a $4.1 \mathrm{mmHg}$ reduction in systolic BP $v$. a $2.0 \mathrm{mmHg}$ reduction among non-drinkers $\left(P_{\text {interaction }}=0.003\right)$ compared to the lowest quintile. The corresponding reductions in diastolic BP were 2.0 v. $1.3 \mathrm{mmHg}\left(P_{\text {interaction }}=0.011\right)$. The 'fruit and milk' pattern was associated with a lower prevalence of both pre-hypertension and hypertension, and the associations appeared to be stronger among drinkers. Results of the present study suggest an important role for diet in the prevention of hypertension.

\section{Dietary patterns: Blood pressure: Hypertension: Chinese men: Alcohol consumption: Smoking}

The prevalence of hypertension, a main contributor to stroke, CHD and early mortality ${ }^{(1)}$, has increased in many countries worldwide, including China. A report from the International Collaborative Study of Cardiovascular Disease in Asia (InterASIA, 2000-2001) ${ }^{(2)}$ showed that the prevalence of hypertension has increased $42 \%$ in Chinese men compared with results from the 1991 Chinese National Hypertension Survey ${ }^{(3)}$. Changes in lifestyle, including diet, and an increase in life expectancy resulting from the recent economic development of China may, in part, explain the rapid increase in the prevalence and absolute number of hypertension cases in China ${ }^{(4)}$.

Many cross-sectional and prospective epidemiological studies have demonstrated that alcohol consumption is one of the most important modifiable risk factors for hypertension among populations from various geographic regions, including North America, Europe and Asia ${ }^{(5-7)}$. Smoking has been shown to have an acute effect on raising blood pressure
(BP) by vasoconstriction and accelerating the heart rate $^{(8-11)}$. The chronic effects of habitual smoking on BP have not been adequately examined in epidemiological studies. A recent meta-analysis of twenty-four case-control studies conducted in China from 1989 to 2001 has suggested that alcohol consumption, smoking, high intake of salt, family history of hypertension, quickness to temper and overweight were the important risk factors for hypertension in China ${ }^{(12)}$.

The role of dietary factors in the modulation of BP among hypertensive and normotensive adults has been investigated in intervention ${ }^{(13,14)}$ and observational studies ${ }^{(15-19)}$. International comparisons and results from studies of migrants and religious groups have suggested that differences in diet may be important determinants of variability in $\mathrm{BP}^{(16)}$. While intervention studies have shown relatively consistent results on dietary intake and $\mathrm{BP}$, the interventions often 
focused on pre-specified diet and often involved short-term exposure. Dietary pattern analysis has the ability to integrate the complex and subtle interactive effects of many dietary exposures and more closely approximates the biological activity of interdependent nutrients in vivo ${ }^{(14,20)}$. However, both factor analysis and cluster analysis, the two most common approaches used to investigate dietary patterns, are considered a posteriori approaches, which generate dietary patterns based on available, empirical data without an a priori hypothesis ${ }^{(20)}$.

We report here on the associations of dietary patterns, selected lifestyle factors, such as cigarette smoking and alcohol consumption, and their interactions with BP among middle-aged and elderly Chinese men in Shanghai who are participants of a large, population-based cohort study and who had reported no history of physician-diagnosed hypertension.

\section{Subjects and methods}

\section{Study population}

This is a cross-sectional analysis of data collected in the baseline survey of the Shanghai Men's Health Study. The Shanghai Men's Health Study is an ongoing, population-based cohort study conducted in eight typical communities of Shanghai, China ${ }^{(21)}$. All male residents who were 40-74 years of age and had no prior history of cancer were eligible for the study. Trained interviewers visited the homes of 83058 eligible men identified through the Shanghai Resident Registry who lived in the study communities during the time the baseline survey was conducted; 61504 men were recruited men into the study between 2002 and 2006. The participation rate was 74.0\%. Reasons for non-participation were refusals $(21 \cdot 1 \%)$, absence during the study period $(3 \cdot 1 \%)$, and other miscellaneous reasons including poor health or hearing problems $(1.8 \%)$. The study protocol was approved by the Institutional Review Boards of each participating institution, and all participants provided written, informed consent.

The baseline survey was completed by in-person interview using a structured questionnaire designed to collect information on demographic characteristics, lifestyle habits, including dietary intake, cigarette smoking and alcohol consumption, medical history and use of medications, including antihypertensives and hormones. The prevalence of hypertension was assessed by the question, 'Have you ever been diagnosed with hypertension by a physician?'

\section{Blood pressure measurement}

At the baseline survey, BP was measured for $98.2 \%$ of participants (n 60401). After the participants sat quietly for more than $5 \mathrm{~min}$, systolic and diastolic BP were taken using an aneroid sphygmomanometer according to a standard protocol $^{(22)}$. Based on the recommendations of the Seventh Report of the Joint National Committee on Prevention, Detection, Evaluation and Treatment of High Blood Pressure (the Joint National Committee 7 full report, accessed on 28 August 2007$)^{(23)}$, we defined pre-hypertension as systolic $\mathrm{BP} \geq 120$ to $<140 \mathrm{mmHg}$ or diastolic $\mathrm{BP} \geq 80$ to
$<90 \mathrm{mmHg}$ and hypertension as systolic $\mathrm{BP} \geq 140$ or diastolic $\mathrm{BP} \geq 90$.

\section{Assessment of dietary intake}

Dietary information was collected via an in-person interview using a validated $\mathrm{FFQ}^{(24)}$. The FFQ included eighty-one food items, which covered $88.8 \%$ of the commonly consumed foods in urban Shanghai. For each food item or food group, participants were asked how frequently (daily, weekly, monthly, annually or never) they consumed the food or food group, followed by a question on the amount of consumption in liang ( 1 liang $=50 \mathrm{~g}$ ) per unit of time over the past 12 months. For seasonal food consumption (mainly fruits and vegetables), an additional question about months of food consumption per year was asked. Our prior investigation of this FFQ found favourable dietary intake estimation characteristics when compared to dietary intake estimated by multiple $24 \mathrm{~h}$ dietary recalls ${ }^{(20)}$. For example, correlation coefficients between the FFQ and averages from $24 \mathrm{~h}$ dietary recalls ranged between 0.59 and 0.66 for macronutrients, 0.41 and 0.59 for most micronutrients, and 0.41 and 0.66 for major food groups.

\section{Dietary pattern derivation}

Dietary patterns were derived using factor analysis ${ }^{(21,25)}$, with eighty-one individual foods or food groups entered into the analysis as the absolute amount of intake in grams per day. The PROC FACTOR procedure in Statistical Analysis Systems (version 9.1; SAS Institute, Cary, NC, USA) was applied to perform the analysis. This procedure uses factor analysis and orthogonal rotation (Quartimax option in Statistical Analysis Systems) to derive non-correlated factors and to render the results more easily interpretable. To determine which number of factors to retain, we examined both the scree plots and the factors themselves to see which set of factors most meaningfully described the distinct food consumption patterns after adjustment for total energy intake. Three major dietary patterns that account for about $41.4 \%$ of the variation of dietary intake were derived ${ }^{(21)}$. Factors were thereby interpreted as dietary patterns and named after the food groups with the highest loading. These loadings can be considered as correlation coefficients between food groups and dietary patterns and take values between -1 and +1 . A factor score was then calculated for each participant for each of the factors, in which the standardised intakes of each of the eighty-one foods or food groups were weighted by their factor loadings and summed. The sums were then standardised again ((score - mean score)/SD of score).

From these analyses, three factors were extracted and factorloading matrices for the three dietary patterns are listed in Appendix 1. The higher the factor loading of a given food item, the greater the contribution of that food item to the specific factor. Dietary pattern I was heavily loaded with vegetables, such as legumes and leafy vegetables and named the 'vegetable' pattern. Pattern II was heavily loaded with fruits and milk and named the 'fruit and milk' pattern. Pattern III was heavily loaded with meat, poultry and organ meat (heart, brain, tongue, intestine, etc.) and named the 'meat' pattern. 


\section{Statistical analysis}

For the present study, we excluded men who reported a history of hypertension ( $n$ 18359), diabetes ( $n$ 3864), CHD ( $n$ 3154) or stroke ( $n$ 536), or who took antihypertensive medication ( $n$ 14160; not mutually exclusive). These exclusions were made because of concerns that dietary practice and BP could be substantially influenced by disease diagnosis and use of medications. In addition, we excluded men with missing BP data ( $n$ 1103) or with extreme total energy intake $(<2092$ and $>16736 \mathrm{~kJ}(<500$ or $>4000 \mathrm{kcal}) / \mathrm{d}$; $n$ 91). After these exclusions, 39252 men remained for the current analysis.

Study participants were categorised into quintiles of dietary factor scores for each dietary pattern, and participants in the lowest quintile were chosen as the reference group. Mean BP differences associated with each category of dietary factor scores were compared with the reference group, and their $95 \%$ CI were estimated using a multiple regression model. Covariates adjusted for included age, BMI, education, income, cigarette smoking, alcohol consumption, weight gain since age 20 and total dietary energy intake. A linear trend test was performed by treating ordinal score variables as continuous variables in the model. We also tested linearity using continuous dietary factor scores. We conducted analyses stratified by cigarette smoking (never, light $(<20$ cigarettes/d) and heavy $(\geq 20$ cigarettes/d)) and alcohol consumption (never, light ( $<7$ times/week) and heavy ( $\geq 7$ times/week)) status to evaluate the potential interactive effect of these variables on dietary patterns with quintile categories. Tests for interaction were performed by introducing a multiplicative interaction term into the main effect models. We also applied a polychotomous logistic regression model to evaluate associations between each dietary pattern and prevalence of pre-hypertension or hypertension. All tests of statistical significance were based on two-sided probability. Statistical analyses were performed with the use of Statistical Analysis Systems software (version 9.1; SAS Institute, Cary, NC, USA).

\section{Results}

Table 1 presents selected characteristics of study participants according to the three derived dietary patterns. The mean age of the study population was 52.5 (SD 8.9) years. Approximately, $22 \%$ of the study participants had attained college or higher education, $65.8 \%$ reported ever smoking and $31.0 \%$ reported regular alcohol consumption (at least 3 times/week for at least 6 months). About $30 \%$ of study participants engaged in regular exercise (once a week at least for 3 months continuously during the 5 years preceding the interview). The mean values of systolic and diastolic BP were 121.9 (SD 15.4) and 79.9 (SD 9.4) $\mathrm{mmHg}$, respectively. The prevalence of pre-hypertension and hypertension were 47.6 and $25.1 \%$, respectively. Men with a higher score for the 'vegetable' pattern were more likely to drink alcohol compared with men with a lower score. Men with a higher score for the 'fruit and milk' pattern were older, had higher educational attainment and higher income, and tended to exercise regularly, but were less likely to smoke cigarettes or drink alcohol than men with a lower score. Men with a higher score for the 'meat' pattern were younger and were more likely to smoke cigarettes and drink alcohol compared to men with a lower score (Table 1). Men with higher scores for either the 'vegetable' or the 'meat' pattern also had higher intakes of $\mathrm{Na}, \mathrm{K}, \mathrm{Ca}$ and $\mathrm{Mg}$.

The associations of cigarette smoking and alcohol consumption with BP are presented in Table 2. Alcohol consumption was positively associated with both systolic and diastolic $\mathrm{BP}$, while cigarette smoking was only associated with systolic BP. The adjusted mean systolic BP was $3.2 \mathrm{mmHg}$ higher (95\% CI 2.9, 3.5) and diastolic BP was $2.0 \mathrm{mmHg}$ higher $(95 \%$ CI $1 \cdot 8,2 \cdot 2)$ for current drinkers compared with never drinkers. The positive association between cigarette smoking or alcohol consumption and BP was statistically stronger among heavy smokers $(P<0.001$, for diastolic BP) and drinkers $(P<0 \cdot 001$, both $\mathrm{BP})$.

After adjustment for confounding factors, the 'fruit and milk' pattern was inversely associated with both systolic and diastolic BP. The adjusted mean systolic BP was $2.9 \mathrm{mmHg}$ lower $(95 \%$ CI $-3.4,-2.4)$ and diastolic BP was $1.7 \mathrm{mmHg}$ lower $(95 \% \mathrm{CI}-2.0,-1.4)$ for men with a score in the highest quintile of the 'fruit and milk' pattern compared with men with a score in the lowest quintile. On the other hand, the 'vegetable' pattern was not associated with either systolic or diastolic BP. The 'meat' pattern was unrelated to systolic BP, but was positively and marginally significantly related to diastolic BP (Table 3 ). The results for all dietary patterns did not change materially when analyses were confined to participants who reported no major changes in their diet during the preceding 5-year period ( $n$ 27 436).

The effect of each dietary pattern on BP was further evaluated by stratifying by alcohol consumption and cigarette smoking status. The inverse association between the 'fruit and milk' pattern and BP was more pronounced among current smokers, particularly among heavy smokers. The interaction test, however, was not statistically significant (Table 4). Among heavy drinkers, on the other hand, the highest quintile of scores for the 'fruit and milk' pattern was associated with $4.1 \mathrm{mmHg}$ lower systolic BP $(95 \% \mathrm{CI}-5 \cdot 2,-3 \cdot 1)$ and $2.0 \mathrm{mmHg}$ lower diastolic BP $(95 \% \mathrm{CI}-2 \cdot 7,-1 \cdot 3)$ compared with the lowest quintile of scores, while the corresponding reductions in systolic and diastolic BP among non-drinkers were $2.0 \mathrm{mmHg}(95 \% \mathrm{CI}-2.6,-1.5)$ and $1.3 \mathrm{mmHg}$ $(95 \%$ CI $-1.6,-0.9)$, respectively (Table 4). Tests for multiplicative interaction were significant for both systolic $(P=0.003)$ and diastolic $(P=0.011) \mathrm{BP}$. The associations of the 'vegetable' and the 'meat' patterns with BP were not modified by smoking or alcohol consumption status (data not shown).

Table 5 presents associations of each dietary pattern with prevalence of pre-hypertension and hypertension based on polychotomous logistic regression analysis stratified by alcohol consumption status. Similar to the results for BP, higher scores for the 'fruit and milk' pattern were associated with a lower prevalence of both pre-hypertension and hypertension, and the associations appeared to be stronger among current drinkers. Although the 'vegetable' and the 'meat' patterns were positively associated with the prevalence of pre-hypertension and hypertension among all participants, the associations were most evident for former alcohol drinkers (Table 5). 
Table 1. Selected characteristics by categories of dietary patterns in the Shanghai Men's Health Study, 2002-6

(Mean values and standard deviations or percentages)

\begin{tabular}{|c|c|c|c|c|c|c|c|c|c|c|c|c|c|c|c|c|c|}
\hline & \multicolumn{2}{|c|}{ All subjects } & \multicolumn{5}{|c|}{ Vegetable dietary pattern } & \multicolumn{5}{|c|}{ Fruit and milk dietary pattern } & \multicolumn{5}{|c|}{ Meat dietary pattern } \\
\hline & \multirow[b]{2}{*}{ Mean } & \multirow[b]{2}{*}{ SD } & \multicolumn{2}{|c|}{ Quintile 1} & \multicolumn{2}{|c|}{ Quintile 5} & \multirow[b]{2}{*}{$P_{\text {trend }}$} & \multicolumn{2}{|c|}{ Quintile 1} & \multicolumn{2}{|c|}{ Quintile 5} & \multirow[b]{2}{*}{$P_{\text {trend }}$} & \multicolumn{2}{|c|}{ Quintile 1} & \multicolumn{2}{|c|}{ Quintile 5} & \multirow[b]{2}{*}{$P_{\text {trend }}$} \\
\hline & & & Mean & SD & Mean & SD & & Mean & SD & Mean & SD & & Mean & SD & Mean & SD & \\
\hline Age (years) & 52.5 & 8.9 & 52.9 & 9.3 & 51.4 & $8 \cdot 3$ & $<0.01$ & $51 \cdot 0$ & 8.0 & 53.6 & 9.5 & $<0.01$ & 53.4 & $9 \cdot 0$ & $50 \cdot 6$ & $8 \cdot 1$ & $<0.01$ \\
\hline $\begin{array}{l}\text { Systolic blood pressure } \\
(\mathrm{mmHg})\end{array}$ & $121 \cdot 9$ & $15 \cdot 4$ & $122 \cdot 1$ & $15 \cdot 9$ & $122 \cdot 4$ & $15 \cdot 2$ & 0.03 & 123.9 & $16 \cdot 4$ & $121 \cdot 1$ & 14.5 & $<0.01$ & 122.5 & $15 \cdot 6$ & $122 \cdot 2$ & $15 \cdot 6$ & 0.80 \\
\hline $\begin{array}{l}\text { Diastolic blood pressure } \\
\quad(\mathrm{mmHg})\end{array}$ & $79 \cdot 9$ & $9 \cdot 4$ & $79 \cdot 8$ & $9 \cdot 6$ & $80 \cdot 4$ & 9.5 & $<0.01$ & $81 \cdot 3$ & $10 \cdot 0$ & $79 \cdot 2$ & $9 \cdot 0$ & $<0.01$ & $79 \cdot 9$ & $9 \cdot 4$ & 80.5 & $9 \cdot 7$ & $<0.01$ \\
\hline $\begin{array}{l}\text { Prevalence of pre- } \\
\text { hypertension (\%) }\end{array}$ & \multicolumn{2}{|c|}{$47 \cdot 6$} & \multicolumn{2}{|c|}{$46 \cdot 4$} & \multicolumn{2}{|c|}{$48 \cdot 6$} & & \multicolumn{2}{|c|}{$45 \cdot 7$} & \multicolumn{2}{|c|}{$49 \cdot 3$} & & \multicolumn{2}{|c|}{$47 \cdot 3$} & \multicolumn{2}{|c|}{$48 \cdot 1$} & \\
\hline $\begin{array}{l}\text { Prevalence of hypertension } \\
(\%)\end{array}$ & \multicolumn{2}{|c|}{$25 \cdot 1$} & \multicolumn{2}{|c|}{$25 \cdot 4$} & & 6.3 & & & & & & & & 5.9 & & 6.2 & \\
\hline Education (\%) & & & & & & & $<0.01$ & & & & & $<0.01$ & & & & & $<0.01$ \\
\hline Elementary school & & 0 & & 4 & & 3 & & & & & & & 6 & .9 & & 4 & \\
\hline Middle school & & $4 \cdot 0$ & 34 & 4.4 & & 2.3 & & & & & & & & 3.1 & & 3.3 & \\
\hline High school & & $9 \cdot 1$ & 39 & $9 \cdot 1$ & & .9 & & & & & & & & 3.4 & & 2.7 & \\
\hline College + & & 1.9 & 20 & 0.1 & & 3.5 & & & & & & & $1 \varepsilon$ & 3.6 & & 0.6 & \\
\hline Income (yuan, \%) & & & & & & & $<0.01$ & & & & & $<0.01$ & & & & & $<0.01$ \\
\hline$<500$ & & 4.4 & 15 & 5.5 & & 4.8 & & & & & & & & 3 & & 6.4 & \\
\hline$>500-1000$ & & $2 \cdot 0$ & & 3.2 & & 9.4 & & & & & & & 45 & 5.6 & & 9.8 & \\
\hline$>1000-2000$ & & $4 \cdot 1$ & & $2 \cdot 3$ & & 5.0 & & & & & & & & 1.3 & & 2.4 & \\
\hline$\geq 2000$ & & .6 & & .0 & & .8 & & & & & & & & 8 & & 1.4 & \\
\hline Smoking (yes, \%) & & $5 \cdot 8$ & & 6.2 & & .0 & 0.16 & & & & & $<0.01$ & & .4 & & 7.9 & $<0.01$ \\
\hline Pack years & $301 \cdot 7$ & $156 \cdot 5$ & $306 \cdot 9$ & $158 \cdot 6$ & 308.5 & $163 \cdot 3$ & 0.01 & 335.9 & 161.5 & 274.7 & 155.5 & $<0.01$ & $279 \cdot 0$ & $149 \cdot 7$ & $341 \cdot 0$ & $169 \cdot 7$ & $<0.01$ \\
\hline $\begin{array}{l}\text { Alcohol consumption* } \\
\text { (ves, \%) }\end{array}$ & & 1.0 & & 2.9 & & 7.9 & $<0.01$ & & & & & $<0.01$ & & 5.3 & & $2 \cdot 1$ & $<0.01$ \\
\hline Amount of alcohol (g/d) & $8 \cdot 7$ & $7 \cdot 6$ & 8.6 & $8 \cdot 1$ & 9.5 & 8.5 & $<0.01$ & $11 \cdot 2$ & $9 \cdot 3$ & $6 \cdot 6$ & $5 \cdot 7$ & $<0.01$ & 7.9 & 7.5 & 10.5 & $8 \cdot 8$ & $<0.01$ \\
\hline Regular exercise (yes, \%) & & 0.0 & & 7.8 & & .8 & $<0.01$ & & & & & $<0.01$ & & 1.1 & & 7.4 & $<0.01$ \\
\hline $\begin{array}{l}\text { LTPA (metabolic equivalent } \\
\text { of task/week) }\end{array}$ & $16 \cdot 0$ & $16 \cdot 2$ & $16 \cdot 7$ & $17 \cdot 3$ & $16 \cdot 2$ & $16 \cdot 1$ & 0.76 & $17 \cdot 6$ & $17 \cdot 8$ & $16 \cdot 2$ & $16 \cdot 3$ & 0.29 & $17 \cdot 9$ & $18 \cdot 1$ & $15 \cdot 7$ & $16 \cdot 7$ & $<0.01$ \\
\hline $\mathrm{BMI}\left(\mathrm{kg} / \mathrm{m}^{2}\right)$ & $23 \cdot 2$ & $3 \cdot 0$ & $23 \cdot 0$ & $3 \cdot 0$ & 23.5 & $3 \cdot 0$ & $<0.01$ & $23 \cdot 3$ & $3 \cdot 0$ & $23 \cdot 3$ & 2.9 & 0.48 & $23 \cdot 4$ & $3 \cdot 0$ & $23 \cdot 0$ & $3 \cdot 0$ & $<0.01$ \\
\hline Waist-hip ratio & 0.89 & 0.06 & 0.89 & 0.06 & 0.89 & 0.05 & $<0.01$ & 0.90 & 0.06 & 0.89 & 0.05 & $<0.01$ & 0.89 & 0.05 & 0.89 & 0.06 & $<0.01$ \\
\hline $\begin{array}{l}\text { Weight gain since age } \\
20 \text { years }(\mathrm{Kg})\end{array}$ & $10 \cdot 6$ & $9 \cdot 3$ & $10 \cdot 0$ & $9 \cdot 4$ & $11 \cdot 2$ & 9.6 & $<0.01$ & $10 \cdot 7$ & 9.6 & $10 \cdot 9$ & $9 \cdot 3$ & $<0.01$ & $11 \cdot 1$ & $9 \cdot 2$ & $10 \cdot 3$ & $9 \cdot 7$ & $<0.01$ \\
\hline Total energy intake $(\mathrm{kJ} / \mathrm{d})$ & 461 & 115 & 504 & 115 & 477 & 120 & 0.81 & 492 & 124 & 479 & 111 & 0.60 & 504 & 105 & 482 & 132 & 0.70 \\
\hline $\mathrm{Na}(\mathrm{mg} / \mathrm{d}) \dagger$ & 2507 & 5979 & 2595 & 9857 & 2698 & 4483 & 0.01 & 2641 & 7779 & 2476 & 2573 & 0.99 & 2433 & 2557 & 2589 & 9988 & $<0.01$ \\
\hline $\mathrm{Ca}(\mathrm{mg} / \mathrm{d})$ & $582 \cdot 7$ & $236 \cdot 6$ & $490 \cdot 0$ & 194.5 & $780 \cdot 7$ & 267.5 & $<0.01$ & 533.7 & 248.4 & $717 \cdot 9$ & 241.0 & $<0.01$ & $561 \cdot 3$ & 233.4 & 679.5 & 269.1 & $<0.01$ \\
\hline $\mathrm{K}(\mathrm{mg} / \mathrm{d})$ & 1932 & 663 & 1651 & 508 & 2578 & 744 & $<0.01$ & 1820 & 667 & 2365 & 669 & $<0.01$ & 1954 & 677 & 2222 & 739 & $<0.01$ \\
\hline$M g(m g / d)$ & 321.9 & $92 \cdot 2$ & $300 \cdot 6$ & $76 \cdot 7$ & 396.0 & $105 \cdot 1$ & $<0.01$ & 328.5 & 97.0 & 359.5 & 96.9 & $<0.01$ & 334.8 & 87.5 & 358.9 & $106 \cdot 7$ & $<0.01$ \\
\hline
\end{tabular}

LTPA, leisure time physical activity.

as 'at least 3 times/week for more than 6 months continuously'.

†Derived from foods, table salt or cooking salt. 


\section{Discussion}

The prevalence of self-reported physician diagnosis of hypertension $(47.9 \%$ for men $40-75$ years of age) in our entire study population is higher than that in the recent InterASIA report (2000-1) on a Chinese population of men $(38.7 \%$ for men $45-75$ years of age $)^{(2)}$. Considering the high prevalence of undiagnosed and untreated hypertension in China ${ }^{(26)}$, the prevalence could be higher. After we excluded men who had a history of physician-diagnosed hypertension and men who had ever used antihypertensive drugs or had a history of diabetes, CHD or stroke at the baseline survey, the prevalence rate of hypertension was still $25 \%$, suggesting a substantial underdiagnosis of hypertension in the present study population. In addition, $47.5 \%$ men had pre-hypertension. In the present study, we found that a dietary pattern with high consumption of fruit and milk was significantly and inversely associated with both lower systolic and diastolic $\mathrm{BP}$ in men. Its effect was more evident among current alcohol drinkers, particularly heavy drinkers, and was independent of other socioeconomic and lifestyle factors. On the other hand, neither a dietary pattern with high consumption of vegetables nor a pattern with high consumption of meat was related to higher systolic or diastolic BP, and no interaction with alcohol consumption was observed.

One aspect of dietary patterns, which is presumably related to BP level, is the co-contributions of micronutrients such as $\mathrm{K}, \mathrm{Ca}, \mathrm{Na}, \mathrm{Mg}$, fibre, etc ${ }^{(27)}$. An alternative explanation points to compounds abundant in fruits and vegetables, such as antioxidants, which help to prevent oxidative stress. For example, it has been shown that $100 \mathrm{~g}$ of fresh apples may have antioxidant activity equivalent to $1500 \mathrm{mg}$ of ascorbic $\operatorname{acid}^{(28)}$. Vascular oxidative stress has been implicated in the pathophysiology of hypertension, resulting in impaired endothelium-dependent vasodilatation ${ }^{(29)}$, although the Supplementation en Vitamines et Minéraux Antioxydants-randomised trial could not demonstrate any beneficial effect of low-dose antioxidant supplementation on 6.5 year risk of hypertension ${ }^{(30)}$.

The Dietary Approaches to Stop Hypertension intervention study $^{(14)}$ and the Oxford Fruit and Vegetable Study ${ }^{(31)}$ have both shown that a diet rich in fruits, vegetables and low-fat dairy products, and low in saturated fats can substantially lower both systolic and diastolic BP. The present finding that a 'fruit and milk' pattern was associated with lower BP is consistent with these reports. However, in the present study, neither the 'vegetable' nor the 'meat' patterns were related to BP. The Coronary Artery Risk Development in Young Adults study reported an inverse association of BP with consumption of a plant-based diet (e.g. whole grains, fruits and nuts) and a positive association with red and processed meat ${ }^{(32)}$. McNaughton et al. ${ }^{(18)}$ reported that a mixed pattern, including fruit, vegetables and dairy products, as well as a meat, potatoes and sweet foods pattern was inversely associated with BP. A higher score for an 'olive oil and vegetables' dietary pattern was associated with lower BP in Italian men ${ }^{(19)}$. A vegetarian diet has been associated with some degree of protection against hypertension compared with a non-vegetarian diet in a Western population ${ }^{(33)}$. On the other hand, a null association between vegetable consumption and BP or hypertension was reported in several other 
Table 3. Association of food groups based on factor analysis with blood pressure (BP) measured at baseline survey of the Shanghai Men's Health Study, 2002-6 (Mean values, standard deviations, OR and $95 \% \mathrm{Cl}$ )

\begin{tabular}{|c|c|c|c|c|c|c|c|c|c|c|c|c|c|}
\hline & \multicolumn{13}{|c|}{ Adjusted difference in BP $(\mathrm{mmHg})^{\star}$ by quintiles of dietary pattern scores for all study subjects ( $n$ 39252) } \\
\hline & \multicolumn{3}{|c|}{ Quintile 1} & \multicolumn{2}{|c|}{ Quintile 2} & \multicolumn{2}{|c|}{ Quintile 3} & \multicolumn{2}{|c|}{ Quintile 4} & \multicolumn{2}{|c|}{ Quintile 5} & \multirow[b]{2}{*}{$P_{\text {trend }}$} & \multirow[b]{2}{*}{$P_{\text {cont }} \dagger$} \\
\hline & OR & Mean $\ddagger$ & SD & $\bar{\chi}$ & $95 \% \mathrm{Cl}$ & $\bar{\chi}$ & $95 \% \mathrm{Cl}$ & $\bar{\chi}$ & $95 \% \mathrm{Cl}$ & $\bar{\chi}$ & $95 \% \mathrm{Cl}$ & & \\
\hline \multicolumn{14}{|l|}{ Systolic BP } \\
\hline Vegetable pattern & 0.0 (reference) & $122 \cdot 1$ & $15 \cdot 9$ & -0.5 & $-1.0,-0.1$ & -0.9 & $-1.4,-0.4$ & -0.7 & $-1 \cdot 2,-0.3$ & -0.0 & $-0.5,0.4$ & 0.624 & 0.287 \\
\hline Fruit and milk pattern & 0.0 (reference) & 123.9 & $16 \cdot 4$ & -1.6 & $-2 \cdot 0,-1 \cdot 1$ & $-2 \cdot 2$ & $-2 \cdot 7,-1 \cdot 8$ & $-3 \cdot 0$ & $-3 \cdot 5,-2 \cdot 6$ & -2.9 & $-3.4,-2 \cdot 4$ & $<0.001$ & $<0.001$ \\
\hline Meat pattern & 0.0 (reference) & $122 \cdot 5$ & $15 \cdot 6$ & -0.5 & $-0.9,-0.0$ & -0.6 & $-1 \cdot 1,-0.2$ & -0.5 & $-1 \cdot 0,-0 \cdot 1$ & 0.2 & $-0.3,0.7$ & 0.514 & 0.133 \\
\hline \multicolumn{14}{|l|}{ Diastolic BP } \\
\hline Vegetable pattern & 0.0 (reference) & $79 \cdot 8$ & $9 \cdot 6$ & -0.2 & $-0.5,0.1$ & -0.5 & $-0.8,-0.2$ & -0.4 & $-0.7,-0.1$ & -0.0 & $-0.3,-0.2$ & 0.474 & 0.574 \\
\hline Fruit and milk pattern & 0.0 (reference) & $81 \cdot 3$ & $10 \cdot 0$ & -0.8 & $-1 \cdot 1,-0.5$ & $-1 \cdot 3$ & $-1 \cdot 6,-1 \cdot 0$ & -1.6 & $-1 \cdot 9,-1 \cdot 3$ & $-1 \cdot 7$ & $-2 \cdot 0,-1 \cdot 4$ & $<0.001$ & $<0.001$ \\
\hline \multirow[t]{4}{*}{ Meat pattern } & 0.0 (reference) & $79 \cdot 9$ & $9 \cdot 4$ & -0.2 & $-0.5,0.1$ & -0.1 & $-0.4,0.1$ & -0.1 & $-0.4,0.2$ & 0.3 & $-0.0,0.6$ & 0.065 & 0.010 \\
\hline & \multicolumn{13}{|c|}{ Adjusted difference in BP $(\mathrm{mmHg})^{*}$ by quintiles of dietary pattern scores for subjects with no major dietary changes $(n 27436$ ) } \\
\hline & \multicolumn{2}{|c|}{ Quintile 2} & & \multicolumn{2}{|c|}{ Quintile 3} & \multicolumn{2}{|r|}{ Quintile 4} & & \multicolumn{2}{|c|}{ Quintile 5} & & \multirow[b]{2}{*}{$P_{\text {cont }} \dagger$} & \\
\hline & $\bar{\chi}$ & $95 \% \mathrm{Cl}$ & & $\bar{\chi}$ & $95 \% \mathrm{Cl}$ & $\bar{\chi}$ & $95 \% \mathrm{Cl}$ & & $\bar{\chi}$ & $95 \% \mathrm{Cl}$ & $P_{\text {trend }}$ & & \\
\hline \multicolumn{14}{|l|}{ Systolic BP } \\
\hline Vegetable pattern & -0.6 & $-1 \cdot 1,-0.1$ & & $-1 \cdot 0$ & $-1.5,-0.5$ & -0.9 & $-1.4,-0.4$ & & -0.3 & $-0 \cdot 8,0.3$ & 0.133 & 0.800 & \\
\hline Fruit and milk pattern & $-1 \cdot 7$ & $-2 \cdot 2,-1 \cdot 1$ & & $-2 \cdot 2$ & $-2 \cdot 8,-1 \cdot 7$ & $-3 \cdot 1$ & $-3 \cdot 6,-2 \cdot 6$ & & -2.9 & $-3 \cdot 4,-2 \cdot 3$ & $<0.001$ & $<0.001$ & \\
\hline Meat pattern & -0.6 & $-1 \cdot 1,-0 \cdot 1$ & & -0.7 & $-1 \cdot 2,-0.2$ & -0.5 & $-1 \cdot 1,0 \cdot 0$ & & 0.1 & $-0 \cdot 4,0 \cdot 7$ & 0.367 & 0.099 & \\
\hline \multicolumn{14}{|l|}{ Diastolic BP } \\
\hline Vegetable pattern & -0.3 & $-0.7,0.0$ & & -0.7 & $-1.0,-0.4$ & -0.6 & $-0.9,-0.2$ & & -0.2 & $-0.5,0.2$ & 0.092 & 0.515 & \\
\hline Fruit and milk pattern & -0.9 & $-1.3,-0.6$ & & $-1 \cdot 3$ & $-1 \cdot 7,-1 \cdot 0$ & -1.6 & $-2 \cdot 0,-1 \cdot 3$ & & -1.6 & $-2 \cdot 0,-1 \cdot 3$ & $<0.001$ & $<0.001$ & \\
\hline Meat pattern & -0.4 & $-0.7,-0.0$ & & -0.3 & $-0.6,0.1$ & -0.2 & $-0.5,0.2$ & & 0.1 & $-0.2,0.5$ & 0.278 & 0.051 & \\
\hline
\end{tabular}

${ }^{*}$ Mean difference in BP with each category of each group from the factor analysis compared with the reference level, estimated by the multiple regression models after adjustment for age, BMI, education, income, cigarette smoking, alcohol consumption, weight gain since age 20 years and total dietary energy intake.

$\dagger$ Test for linearity.

$\ddagger B P$ of men in lowest quintile for each dietary pattern. 
Table 4. Fruit and milk dietary pattern loading score and blood pressure (BP) measured at baseline survey, stratified by smoking and alcohol consumption status, Shanghai Men's Health Study, 2002-6 $(\mathrm{OR}$ and $95 \% \mathrm{Cl})$

\begin{tabular}{|c|c|c|c|c|c|c|c|c|c|c|c|c|c|c|c|c|c|}
\hline & & & & & & \multicolumn{12}{|c|}{ Adjusted difference in $\mathrm{BP}^{*}$ by fruit and milk diet loading score } \\
\hline & \multicolumn{5}{|c|}{ Number of participants } & \multicolumn{2}{|c|}{ Quintile 1} & \multicolumn{2}{|c|}{ Quintile 2} & \multicolumn{2}{|c|}{ Quintile 3} & \multicolumn{2}{|c|}{ Quintile 4} & \multicolumn{2}{|c|}{ Quintile 5} & \multirow[b]{2}{*}{$P_{\text {trend }}$} & \multirow[b]{2}{*}{$P_{\text {interactior }}$} \\
\hline & $\begin{array}{c}\text { Quintile } \\
1\end{array}$ & $\begin{array}{c}\text { Quintile } \\
2\end{array}$ & $\begin{array}{c}\text { Quintile } \\
3 \\
\end{array}$ & $\begin{array}{c}\text { Quintile } \\
4\end{array}$ & $\begin{array}{c}\text { Quintile } \\
5\end{array}$ & OR & $95 \% \mathrm{Cl}$ & OR & $95 \% \mathrm{Cl}$ & OR & $95 \% \mathrm{Cl}$ & OR & $95 \% \mathrm{Cl}$ & OR & $95 \% \mathrm{Cl}$ & & \\
\hline \multicolumn{18}{|l|}{$\begin{array}{l}\text { Cigarette smoking } \\
\text { Systolic BP }\end{array}$} \\
\hline Never & 1031 & 1444 & 2076 & 2060 & 3138 & 0.0 & Ref. & -0.7 & $-1.8,0.4$ & $-1 \cdot 1$ & $-2 \cdot 2,-0 \cdot 1$ & $-2 \cdot 0$ & $-3 \cdot 1,-1 \cdot 0$ & $-2 \cdot 2$ & $-3 \cdot 2,-1 \cdot 2$ & $<0.001$ & \\
\hline Formert & 438 & 566 & 670 & 720 & 756 & 0.0 & Ref. & -1.6 & $-3.5,0.3$ & $-2 \cdot 0$ & $-3 \cdot 9,-0 \cdot 2$ & -3.4 & $-5 \cdot 2,-1 \cdot 5$ & -3.1 & $-4 \cdot 9,-1 \cdot 2$ & $<0.001$ & \\
\hline Current & 6420 & 5871 & 5140 & 4495 & 3880 & 0.0 & Ref. & $-2 \cdot 1$ & $-2 \cdot 6,-1 \cdot 6$ & -3.0 & $-3 \cdot 5,-2 \cdot 5$ & -3.8 & $-4 \cdot 3,-3 \cdot 2$ & $-3 \cdot 6$ & $-4 \cdot 2,-3 \cdot 0$ & $<0.001$ & $0.828 \ddagger$ \\
\hline$<20($ cigarettes/d) & 5468 & 5195 & 4643 & 4139 & 3567 & 0.0 & Ref. & -1.5 & $-2 \cdot 2,-0.7$ & -2.5 & $-3 \cdot 3,-1 \cdot 7$ & -3.1 & $-3 \cdot 9,-2 \cdot 3$ & $-2 \cdot 4$ & $-3 \cdot 2,-1 \cdot 6$ & $<0.001$ & \\
\hline$\geq 20($ cigarettes $/ \mathrm{d})$ & 952 & 676 & 497 & 356 & 313 & 0.0 & Ref. & -2.5 & $-3 \cdot 2,-1 \cdot 8$ & $-3 \cdot 2$ & $-3 \cdot 9,-2 \cdot 5$ & $-4 \cdot 1$ & $-4 \cdot 9,-3 \cdot 3$ & -4.7 & $-5 \cdot 6,-3 \cdot 9$ & $<0.001$ & $0.748 \S$ \\
\hline \multicolumn{18}{|l|}{ Diastolic BP } \\
\hline Never & 1031 & 1444 & 2076 & 2060 & 3138 & 0.0 & Ref. & -0.4 & $-1 \cdot 1,0.3$ & -0.6 & $-1 \cdot 2,0 \cdot 1$ & -0.9 & $-1 \cdot 5,-0.2$ & $-1 \cdot 2$ & $-1.8,-0.5$ & $<0.001$ & \\
\hline Formert & 438 & 566 & 670 & 720 & 756 & 0.0 & Ref. & -0.5 & $-1.6,0.7$ & -0.7 & $-1.8,0.4$ & -1.4 & $-2.5,-0.3$ & -1.2 & $-2 \cdot 2,0 \cdot 0$ & 0.017 & \\
\hline Current & 6420 & 5871 & 5140 & 4495 & 3880 & 0.0 & Ref. & $-1 \cdot 1$ & $-1.4,-0.8$ & $-1 \cdot 8$ & $-2 \cdot 2,-1 \cdot 5$ & $-2 \cdot 1$ & $-2 \cdot 5,-1 \cdot 8$ & $-2 \cdot 2$ & $-2 \cdot 6,-1 \cdot 8$ & $<0.001$ & 0.675 \\
\hline$<20($ cigarettes/d) & 5468 & 5195 & 4643 & 4139 & 3567 & 0.0 & Ref. & -0.7 & $-1 \cdot 2,-0.2$ & $-1 \cdot 7$ & $-2 \cdot 2,-1 \cdot 2$ & -1.9 & $-2 \cdot 4,-1.4$ & $-1 \cdot 7$ & $-2 \cdot 3,-1 \cdot 2$ & $<0.001$ & \\
\hline$\geq 20$ (cigarettes/d) & 952 & 676 & 497 & 356 & 313 & 0.0 & Ref. & -1.3 & $-1 \cdot 8,-0.9$ & $-1 \cdot 8$ & $-2 \cdot 3,-1 \cdot 4$ & $-2 \cdot 2$ & $-2 \cdot 7,-1 \cdot 6$ & -2.6 & $-3 \cdot 1,-2 \cdot 0$ & $<0.001$ & 0.332 \\
\hline \multicolumn{18}{|l|}{ Alcohol consumption\| } \\
\hline \multicolumn{18}{|l|}{ Systolic BP } \\
\hline Never & 4254 & 4848 & 5289 & 5575 & 5955 & 0.0 & Ref. & $-1 \cdot 0$ & $-1.5,-0.4$ & -1.4 & $-1 \cdot 9,-0 \cdot 8$ & $-2 \cdot 2$ & $-2 \cdot 8,-1 \cdot 7$ & -2.0 & $-2 \cdot 6,-1 \cdot 5$ & $<0.001$ & \\
\hline Formerף & 287 & 239 & 207 & 205 & 216 & 0.0 & Ref. & $-1 \cdot 3$ & $-3 \cdot 8,1 \cdot 3$ & $-2 \cdot 2$ & $-4 \cdot 8,0.5$ & $-1 \cdot 8$ & $-4.5,0.9$ & -3.8 & $-6 \cdot 5,-1 \cdot 1$ & 0.009 & \\
\hline Current & 3348 & 2794 & 2390 & 2041 & 1603 & 0.0 & Ref. & -2.5 & $-3 \cdot 2,-1 \cdot 7$ & -3.6 & $-4 \cdot 4,-2 \cdot 8$ & $-4 \cdot 2$ & $-5 \cdot 0,-2 \cdot 4$ & $-5 \cdot 3$ & $-7 \cdot 1,-3 \cdot 5$ & $<0.001$ & 0.004 \\
\hline$<7$ (times/week) & 2801 & 2432 & 2136 & 1849 & 1449 & 0.0 & Ref. & -3.4 & $-5 \cdot 0,-1 \cdot 8$ & -3.4 & $-5 \cdot 1,-1 \cdot 7$ & $-4 \cdot 1$ & $-5 \cdot 8,-2 \cdot 4$ & $-5 \cdot 3$ & $-7 \cdot 1,-3 \cdot 5$ & $<0.001$ & \\
\hline$\geq 7$ (times/week) & 547 & 362 & 252 & 191 & 154 & 0.0 & Ref. & $-2 \cdot 2$ & $-3 \cdot 1,-1 \cdot 4$ & -3.6 & $-4 \cdot 5,-2 \cdot 7$ & $-4 \cdot 1$ & $-5 \cdot 1,-3 \cdot 2$ & $-4 \cdot 1$ & $-5 \cdot 2,-3 \cdot 1$ & $<0.001$ & 0.003 \\
\hline \multicolumn{18}{|l|}{ Diastolic BP } \\
\hline Never & 4254 & 4848 & 5289 & 5575 & 5955 & 0.0 & Ref. & -0.6 & $0.9,-0.2$ & -0.9 & $-1.2,-0.5$ & $-1 \cdot 1$ & $-1.5,-0.8$ & $-1 \cdot 3$ & $-1.6,-0.9$ & $<0.001$ & \\
\hline Formerq & 287 & 239 & 207 & 205 & 216 & 0.0 & Ref. & -0.1 & $-1 \cdot 6,1 \cdot 5$ & -0.8 & $-2.4,0.9$ & -0.0 & $-1 \cdot 7,1 \cdot 7$ & -1.8 & $-3.5,-0.2$ & 0.061 & \\
\hline Current & 3348 & 2794 & 2390 & 2041 & 1603 & 0.0 & Ref. & $-1 \cdot 2$ & $-1.7,-0.7$ & $-2 \cdot 0$ & $-2 \cdot 5,-1.5$ & -2.4 & $-3 \cdot 0,-1 \cdot 3$ & -3.0 & $-4 \cdot 2,-1 \cdot 8$ & $<0.001$ & 0.038 \\
\hline$<7$ (times/week) & 2801 & 2432 & 2136 & 1849 & 1449 & 0.0 & Ref. & -0.9 & $-1 \cdot 9,0 \cdot 1$ & $-1 \cdot 6$ & $-2 \cdot 7,-0.5$ & $-2 \cdot 4$ & $-3 \cdot 6,-1 \cdot 3$ & -3.0 & $-4 \cdot 2,-1 \cdot 8$ & $<0.001$ & \\
\hline$\geq 7$ (times/week) & 547 & 362 & 252 & 191 & 154 & 0.0 & Ref. & $-1 \cdot 3$ & $-1.8,-0.7$ & $-2 \cdot 0$ & $-2 \cdot 6,-1 \cdot 5$ & $-2 \cdot 4$ & $-3 \cdot 0,-1 \cdot 8$ & -2.0 & $-2 \cdot 7,-1 \cdot 3$ & $<0.001$ & 0.011 \\
\hline
\end{tabular}

\section{Ref., reference.}

${ }^{*}$ Mean difference in BP with each category of each group from the factor analysis compared with the reference level, estimated by the multiple regression models after adjustment for age, BMI, education, income, weight gain since age 20 years, total dietary energy intake, and total intake of $\mathrm{Na}$ and $\mathrm{Ca}$.

† Former smokers included men who had ever smoked at least 1 cigarette/d for more than 6 months but were not smoking at the time of the interview. $<20$ (cigarettes/d) $v$. $\geq 20$ (cigarettes/d): smoked $<20$ cigarettes/d $v$. $>20$ cigarettes/d. $\ddagger P$ for interaction between smoking or alcohol consumption (never and ever smoker/drinker) and five categories of the fruit and milk diet.

$\S P$ for interaction between smoking or alcohol consumption (no currently, light and heavy smoker/drinker) and five categories of fruit and milk diet.

Alcohol consumption was defined as 'at least 3 times/week for more than 6 months continuously'.

ๆ Former drinkers included men who had ever drunk alcohol at least 3 times/week for more than 6 months continuously, but were not drinking at the time of the interview. $<7$ (times/week) $v$. $\geq 7$ (times/week): drunk $<7$ times/week $v$. $>7$ times/week. 
Table 5. Association of dietary patterns with pre-hypertension and hypertension according to polychotomous multiple regression analysis stratified by alcohol consumption* (OR and $95 \% \mathrm{Cl}$ )

\begin{tabular}{|c|c|c|c|c|c|c|c|c|c|c|c|c|c|c|c|c|c|c|c|}
\hline & \multicolumn{4}{|c|}{ All subjects ( $n 39291$ ) } & \multicolumn{5}{|c|}{ Non-drinker ( $n$ 25953) } & \multicolumn{5}{|c|}{ Former drinker $(n 1146)$} & \multicolumn{5}{|c|}{ Current drinker ( $n$ 12 191) } \\
\hline & All & \multicolumn{2}{|c|}{$\begin{array}{l}\text { Pre-hypertensive } \\
\text { ( } n \text { 18697) }\end{array}$} & $\begin{array}{l}\text { Hypertensive } \\
\text { (n 9853) }\end{array}$ & \multicolumn{2}{|r|}{ All } & \multicolumn{2}{|c|}{$\begin{array}{l}\text { Pre-hypertensive } \\
\qquad(n \text { 18697) }\end{array}$} & $\begin{array}{l}\text { Hypertensive } \\
\text { (n 9853) }\end{array}$ & \multicolumn{2}{|r|}{ All } & \multicolumn{2}{|c|}{$\begin{array}{l}\text { Pre-hypertensive } \\
\qquad(n \text { 18697) }\end{array}$} & $\begin{array}{l}\text { Hypertensive } \\
\text { (n 9853) }\end{array}$ & \multicolumn{2}{|r|}{ All } & \multicolumn{2}{|c|}{$\begin{array}{l}\text { Pre-hypertensive } \\
\text { ( } n \text { 18697) }\end{array}$} & $\begin{array}{l}\text { Hypertensive } \\
\text { (n 9853) }\end{array}$ \\
\hline & $\mathrm{OR} \quad 95 \% \mathrm{Cl}$ & OR & $95 \% \mathrm{Cl}$ & $\mathrm{OR} \quad 95 \% \mathrm{Cl}$ & OR & $95 \% \mathrm{Cl}$ & OR & $95 \% \mathrm{Cl}$ & OR $95 \% \mathrm{Cl}$ & $\mathrm{OR}$ & $95 \% \mathrm{Cl}$ & OR & $95 \% \mathrm{Cl}$ & $\mathrm{OR} \quad 95 \% \mathrm{Cl}$ & OR & $95 \% \mathrm{Cl}$ & OR & $95 \% \mathrm{Cl}$ & $\mathrm{OR} \quad 95 \% \mathrm{Cl}$ \\
\hline \multicolumn{20}{|c|}{ Vegetable pattern } \\
\hline Q1 & Reference & \multicolumn{2}{|c|}{ Reference } & \multirow{2}{*}{$\begin{array}{c}\text { Reference } \\
0.950 .86,1.04\end{array}$} & \multirow{2}{*}{\multicolumn{2}{|c|}{$\begin{array}{l}\text { Reference } \\
\text { Rere }\end{array}$}} & \multicolumn{2}{|c|}{ Reference } & \multirow{2}{*}{$\begin{array}{c}\text { Reference } \\
0.910 .82,1.02\end{array}$} & \multirow{2}{*}{\multicolumn{2}{|c|}{$\begin{array}{c}\text { Reference } \\
1.270 .84,1.94\end{array}$}} & \multicolumn{2}{|c|}{ Reference } & \multirow{2}{*}{$\begin{array}{c}\text { Reference } \\
1.310 .79,2.18\end{array}$} & \multirow{2}{*}{\multicolumn{2}{|c|}{$\begin{array}{c}\text { Reference } \\
0.940 .81,1 \cdot 10\end{array}$}} & \multicolumn{2}{|c|}{ Reference } & Reference \\
\hline Q2 & $0.980 .91,1.06$ & 1.00 & $0.93,1.08$ & & & & 0.99 & $0.91,1.09$ & & & & 1.24 & $0.79,1.95$ & & & & 0.95 & $0.81,1 \cdot 12$ & $0.940 .78,1 \cdot 12$ \\
\hline Q3 & $1.000 .93,1.07$ & 1.03 & $0.95,1.11$ & $0.940 .85,1.03$ & 0.95 & $0.87,1.04$ & 0.98 & $0.90,1.08$ & $0.880 .79,0.99$ & 1.60 & $1.04,2.45$ & 1.79 & $1 \cdot 14,2 \cdot 82$ & $1.280 .76,2.17$ & 0.97 & $0.84,1.13$ & 1.00 & $0.85,1 \cdot 17$ & $0.930 .77,1.11$ \\
\hline Q4 & $1.030 .96,1.11$ & 1.07 & $0.99,1.15$ & $0.970 .88,1.06$ & 0.96 & $0.88,1.05$ & 1.02 & $0.93,1.12$ & $0.840 .75,0.94$ & 1.59 & $1.02,2.47$ & 1.61 & $1.01,2.58$ & $1.560 .92,2.65$ & 1.01 & $0.89,1.17$ & 1.02 & $0.87,1.19$ & $1.000 .85,1 \cdot 17$ \\
\hline Q5 & $1.151 .07,1.24$ & $1 \cdot 15$ & $1.06,1.25$ & $1.141 .04,1.25$ & 1.06 & $0.97,1 \cdot 16$ & 1.09 & $0.99,1.20$ & $0.990 .88,1.11$ & 1.72 & $1.11,2.66$ & 1.64 & $1.03,2.62$ & $1.861 \cdot 10,3 \cdot 13$ & 1.08 & $0.93,1.25$ & 1.07 & $0.92,1.25$ & $1.090 .91,1.29$ \\
\hline$P_{\text {trend }}$ & 0.0001 & & .0001 & 0.0056 & & 3510 & & 0880 & 0.3334 & & 0069 & & 0.143 & 0.0147 & & .1247 & & 1738 & 0.1411 \\
\hline Fruit an & milk pattern & & & & & & & & & & & & & & & & & & \\
\hline Q1 & Reference & & ference & Reference & & ference & & ference & Reference & & ference & & ference & Reference & & ference & & ference & Reference \\
\hline Q2 & $0.840 .78,0.91$ & 0.90 & $0.83,0.97$ & $0.930 .68,0.81$ & 0.93 & $0.84,1.03$ & 0.96 & $0.84,1.03$ & $0.850 .76,0.97$ & 0.95 & $0.63,1.44$ & 0.98 & $0.63,1.54$ & $0.900 .55,1.48$ & 0.78 & $0.68,0.88$ & 0.84 & $0.73,0.96$ & $0.680 .59,0.79$ \\
\hline Q3 & $0.780 .72,0.84$ & 0.85 & $0.79,0.93$ & $0.630 .57,0.69$ & 0.89 & $0.81,0.98$ & 0.94 & $0.81,0.98$ & $0.770 .68,0.87$ & 0.88 & $0.57,1.36$ & 0.98 & $0.62,1.55$ & $0.740 .44,1.24$ & 0.68 & $0.60,0.78$ & 0.77 & $0.67,0.89$ & $0.550 .47,0.64$ \\
\hline Q4 & $0.760 .70,0.82$ & 0.87 & $0.80,0.94$ & $0.570 .51,0.62$ & 0.85 & $0.77,0.94$ & 0.92 & $0.83,1.02$ & $0.690 .61,0.78$ & 1.15 & $0.73,1.83$ & 1.24 & $0.76,2.03$ & $1.010 .59,1.74$ & 0.73 & $0.63,0.84$ & 0.88 & $0.76,1.02$ & $0.500 .42,0.59$ \\
\hline Q5 & $0.740 .68,0.80$ & 0.84 & $0.78,0.92$ & $0.550 .50,0.61$ & 0.84 & $0.76,0.92$ & 0.91 & $0.82,1.01$ & $0.670 .59,0.76$ & 0.80 & $0.51,1.23$ & 0.92 & $0.58,1.46$ & $0.600 .35,1.03$ & 0.71 & $0.61,0.83$ & 0.83 & $0.71,0.98$ & $0.530 .44,0.64$ \\
\hline$P_{\text {trend }}$ & $<0.0001$ & & 0.0001 & $<0.0001$ & & 0.0001 & & 0.0498 & $<0.0001$ & & .5582 & & .9588 & 0.1324 & & 0.0001 & & .0324 & $<0.0001$ \\
\hline Meat $p$ & tern & & & & & & & & & & & & & & & & & & \\
\hline Q1 & Reference & & ference & Reference & & ference & & ference & Reference & & ference & & ference & Reference & & ference & & ference & Reference \\
\hline Q2 & $1.000 .93,1.08$ & 1.01 & $0.94,1 \cdot 10$ & $0.970 .88,1.06$ & 0.99 & $0.91,1.07$ & 1.00 & $0.92,1.09$ & $0.960 .86,1.07$ & 1.27 & $0.83,1.94$ & $1 \cdot 30$ & $0.83,2.04$ & $1.210 .72,2.02$ & 0.98 & $0.82,1 \cdot 18$ & 1.00 & $0.82,1.22$ & $0.950 .77,1.19$ \\
\hline Q3 & $1.020 .95,1 \cdot 10$ & 1.04 & $0.96,1 \cdot 12$ & $1.000 .91,1.10$ & 1.00 & $0.91,1 \cdot 10$ & 1.06 & $0.97,1 \cdot 16$ & $0.960 .86,1.07$ & $1 \cdot 10$ & $0.72,1.67$ & 1.00 & $0.63,1.57$ & $1 \cdot 280.77,2 \cdot 12$ & 0.90 & $0.75,1.07$ & 0.89 & $0.74,1.08$ & $0.900 .73,1 \cdot 11$ \\
\hline Q4 & $1.070 .99,1.15$ & 1.07 & $0.99,1.16$ & $1.050 .96,1.16$ & 1.00 & $0.91,1.09$ & 1.02 & $0.93,1.12$ & $0.950 .84,1.06$ & 1.01 & $0.66,1.54$ & 1.03 & $0.66,1.61$ & $0.990 .59,1.65$ & 1.00 & $0.84,1.19$ & 1.01 & $0.85,1.22$ & $0.970 .79,1.19$ \\
\hline Q5 & $1.281 \cdot 19,1.38$ & 1.24 & $1 \cdot 15,1 \cdot 35$ & $1.371 .25,1.50$ & 1.12 & $1.02,1.24$ & $1 \cdot 16$ & $1 \cdot 05,1 \cdot 28$ & $1.040 .92,1.18$ & 1.37 & $0.88,2 \cdot 12$ & 1.33 & $0.84,2 \cdot 13$ & $1.430 .85,2.43$ & 1.09 & $0.93,1.28$ & 1.06 & $0.89,1.26$ & $1.150 .95,1.39$ \\
\hline$P_{\text {trend }}$ & $<0.0001$ & & 0.0001 & $<0.0001$ & & 0.048 & & 0117 & 0.8566 & & .4145 & & .5184 & 0.3765 & & .0564 & & 1781 & 0.0181 \\
\hline
\end{tabular}

* OR adjusted for age, BMI, education, income, cigarette smoking, weight gain since age 20 years and total dietary energy intake compared to subjects with normal blood pressure. 
clinical interventions $^{(30)}$ and observational ${ }^{(17,32,34)}$ studies. It is noteworthy that the definition of the 'vegetable' pattern in the present study is different from that used in the Dietary Approaches to Stop Hypertension study and other studies. In addition, because many lifestyle factors are associated with dietary patterns and there is overlap between dietary patterns, it is difficult to truly distinguish one dietary pattern from another.

There are several possible explanations for the different associations observed for the 'vegetable' and the 'fruit and milk' patterns in the present study. First, a high score for the 'vegetable' or the 'meat' pattern was related to a high intake of $\mathrm{Na}$, while a high score for the 'fruit and milk' pattern was not related to a dietary $\mathrm{Na}$ intake. Intake of total $\mathrm{Na}$ was positively associated with both systolic and diastolic BP in the present study population, consistent with results from other populations ${ }^{(27,34)}$. However, additional adjustment for $\mathrm{Na}$ and $\mathrm{Ca}$ intakes did not change the associations of dietary patterns with BP. In contrast to the eating habits of Western populations, which often consume vegetables that are raw and fresh, Chinese populations tend to eat vegetables that have been cooked with salt or that have been pickled. Second, mineral absorption in the intestine is affected by compounds that are consumed at the same time and interact with other minerals. For instance, phytate and oxalate, both abundant in vegetables, can impair the bioavailability of $\mathrm{Ca}$, $\mathrm{Fe}$ and $\mathrm{Zn}$, and phytate content depends to some extent on food processing and cooking methods ${ }^{(35)}$. On the other hand, the citric and ascorbic acids, abundant in fruit, have been reported to have a synergistic effect on the mineral absorption and bioavailability of $\mathrm{Ca}$ and $\mathrm{P}$ in the body ${ }^{(36)}$. Third, in view of the effect of antioxidants on BP, cooking vegetables before they are eaten may result in the loss of some antioxidant content (e.g. vitamin C). Finally, because the association of the 'vegetable' pattern with BP was no longer present when the analysis was restricted to participants who reported no major changes in recent diet, reverse causality, i.e. diet modification as a means to prevent high BP among men with high BP, could not be excluded.

The positive association between alcohol consumption and $\mathrm{BP}$ is widely recognised. Consistent with the present results, many epidemiological studies have reported a positive association between alcohol consumption and $\mathrm{BP}^{(37-39)}$. A prospective cohort study in Japan ${ }^{(40)}$ observed that the average annual increase in systolic BP was greater among alcohol drinkers who consumed $\geq 300 \mathrm{~g} /$ week than among nondrinkers, suggesting a hypertensive effect of long-term alcohol consumption. Smoking causes an acute increase in BP and heart rate and possibly malignant hypertension ${ }^{(41)}$, which could be explained by nicotine acting as an adrenergic agonist, mediating local and systemic catecholamine release of vaso$\operatorname{pressin}^{(11)}$. A study in England showed a small independent effect of smoking on $\mathrm{BP}^{(42)}$, similar to results from the present study. However, a cross-sectional study in Japan found lower $\mathrm{BP}$ in cigarette smokers ${ }^{(43)}$. It is noteworthy that in the present study, the association of the 'fruit and milk' pattern with BP was modified by alcohol consumption status; the association between the 'fruit and milk' pattern and BP was stronger among current and heavy alcohol drinkers. To our knowledge, no study has reported a combined effect of dietary patterns and alcohol consumption on BP, although Criqui et al. ${ }^{(44)}$ reported that intakes of $\mathrm{Ca}$ and $\mathrm{K}$ were significantly and inversely related to $\mathrm{BP}$ in non-drinkers and light drinkers compared with heavy drinkers. The present study appears to suggest that the nutrients, including antioxidants and certain minerals abundant in the 'fruit and milk' pattern, may counteract the negative effects of alcohol consumption that cause vascular damage. More studies are needed to confirm the present findings.

It is noteworthy that given the cross-sectional nature of the present study, no causal association of dietary patterns with BP can be established. Although careful adjustment for multiple confounders did not appreciably change the results, we could not completely exclude the possibility of residual confounding due to unmeasured or inaccurately measured covariates, such as information on family history of hypertension and BP-related diseases including hypercholesterolaemia. It is possible that men with a known family history of hypertension or with hypercholesterolaemia were more likely to pursue healthier lifestyles and dietary practices than those without such a family history or condition. BMI and weight gain are related to BP and dietary patterns, and thus may act as confounders. It is also possible they are in the causal pathway. In the present study, the association of the 'fruit and milk' pattern with BP changed little with or without adjustment for BMI and weight gain (data not shown). The 'vegetable' pattern, on the other hand, was inversely associated with BP without adjustment for BMI and weight gain, indicating possible overadjustment (data not shown). However, we also found that the 'vegetable' pattern was positively associated with BMI and weight gain, suggesting possible reverse causation (data not shown). Studies with a prospective design are needed to disentangle the nature of the relationship between dietary patterns and BP. Because there are many potential differences in nutrients between dietary patterns, this approach cannot determine the specific nutrients responsible for $\mathrm{BP}$ differences. Dietary patterns are likely to vary according to sex, socioeconomic status, ethnic group and culture, and the meaning of a dietary pattern could change over time because of changes in food preferences and food availability ${ }^{(20)}$. Although the distribution of age, sex, education level and occupation in the eight participant communities is similar to the general population of urban Shanghai, it is unclear whether the present findings can be generalised to residents of sub-urban Shanghai or to other cities in China. Thus, it is necessary to replicate these results in diverse populations. Nevertheless, the present study has several strengths. The population-based study design and high response rate minimised selection bias. BP was measured in participants' homes by trained medical professionals. The comprehensive information on lifestyle and dietary factors allowed for adjustment of a broad range of potential confounding variables.

In summary, we found that the 'fruit and milk' pattern was inversely associated with $\mathrm{BP}$, and the effect was more pronounced in current and heavy alcohol drinkers. The present results suggest that modifying dietary practice may be an effective means of combating high BP.

\section{Acknowledgements}

S.-A. L. drafted the manuscript and analysed the data. X. O. S. and W. Z. designed and obtained the funding for the 
overall study. X. O. S., G. Y., Y.-B. X., H. L., W.-H. X. and Y.-T. G. directed and supervised the field operation and data cleaning. X. O. S., G. Y. and W. Z. provided critical advice in data analysis and manuscript preparation. H. C. contributed to the statistical analysis. All co-authors have reviewed and approved the paper. The present study was supported by US PHS grant number R01 CA82729 from the National Cancer Institute. The authors have no conflicts of interest to declare.

\section{References}

1. Lewington S, Clarke R, Qizilbash N, et al. (2002) Age-specific relevance of usual blood pressure to vascular mortality: a meta-analysis of individual data for one million adults in 61 prospective studies. Lancet 360, 1903-1913.

2. Gu D, Reynolds K, Wu X, et al. (2002) Prevalence, awareness, treatment, and control of hypertension in China. Hypertension 40, 920-927.

3. Wu X, Duan X, Gu D, et al. (1995) Prevalence of hypertension and its trends in Chinese populations. Int J Cardiol 52, 39-44.

4. Chen J (1999) Dietary changes and disease transition in China (Review). Nutrition 15, 330-331.

5. He J \& Bazzano LA (2000) Effects of lifestyle modification on treatment and prevention of hypertension. Curr Opin Nephrol Hypertens 9, 267-271.

6. Keil U, Liese A, Filipiak B, et al. (1998) Alcohol, blood pressure and hypertension. Novartis Found Symp 216, 1157-1170.

7. Beilin LJ \& Puddey IB (1993) Alcohol, hypertension and cardiovascular disease - implications for management. Clin Exp Hypertens 15, 1157-1170.

8. Benowitz NL, Kuyt F \& Jacob P 3rd (1984) Influence of nicotine on cardiovascular and hormonal effects of cigarette smoking. Clin Pharmacol Ther 36, 74-81.

9. Aronow WS, Dendinger J \& Rokaw SN (1971) Heart rate and carbon monoxide level after smoking high-, low-, and non-nicotine cigarettes. A study in male patients with angina pectoris. Ann Intern Med 74, 697-702.

10. Benowitz NL, Jacob P 3rd, Jones RT, et al. (1982) Interindividual variability in the metabolism and cardiovascular effects of nicotine in man. J Pharmacol Exp Ther 221, 368-372.

11. Cryer PE, Haymond MW, Santiago JV, et al. (1976) Norepinephrine and epinephrine release and adrenergic mediation of smoking-associated hemodynamic and metabolic events. $N$ Engl J Med 295, 573-577.

12. Luo L, Luan RS \& Yuan P (2003) Meta-analysis of risk factors on hypertension in China. Zhanghua Liu Xing Bing Xue Za Zhi 24, 50-53.

13. Svetkey LP, Simons-Morton D, Vollmer WM, et al. (1999) Effects of dietary patterns on blood pressure: subgroup analysis of the dietary approaches to stop hypertension (DASH) randomized clinical trial. Arch Intern Med 159, 285-293.

14. Appel LJ, Moore TJ, Obarzanek E, et al. (1997) A clinical trial of the effects of dietary patterns on blood pressure. N Engl J Med 336, $1117-1124$

15. Miura K, Greenland P, Stamler J, et al. (2004) Relation of vegetable, fruit, and meat intake to 7-year blood pressure change in middle-aged men: the Chicago Western Electric Study. Am J Epidemiol 159, 572-580.

16. Ascherio A, Stampfer MJ, Colditz GA, et al. (1991) Nutrient intakes and blood pressure in normotensive males. Int J Epidemiol 20, 886-891.
17. Sanakane A, Teutsumi A, Gotoh T, et al. (2008) Dietary patterns and levels of blood pressure and serum lipids in a Japanese population. J Epidemiol 18, 58-67.

18. McNaughton SA, Mishra GD, Stephen AM, et al. (2007) Dietary patterns throughout adult life are associated with body mass index, waist circumference, blood pressure, and red cell folate. J Nutr 137, 99-105.

19. Centritto F, Lacoviello L, di Giusepe R, et al. (2009) Dietary patterns, cardiovascular risk factors and C-reactive protein in a healthy Italian population. Nuri Metab Cardiovasc Dis (Epublication ahead of print version).

20. Hu FB (2002) Dietary pattern analysis: a new direction in nutritional epidemiology. Curr Opin Lipidol 13, 3-9.

21. Cai H, Zheng W, Xiang YB, et al. (2007) Dietary patterns and their correlates among middle-aged and elderly Chinese men: a report from the Shanghai Men's Health Study. Br J Nutr 98, 1006-1013.

22. Perloff D, Grim C, Flack J, et al. (1993) Human BP determination by sphygmomanometer. Circulation $\mathbf{8 8}$, $2460-2470$.

23. National Heart, Lung and Blood Institute. The Seventh Report of the Joint National Committee on Prevention, Detection, Evaluation, and Treatment of High Blood Pressure Complete Report. http://www.nhlbi.nih.gov/guidelines/hyperten sion/jnc7full.htm

24. Villegas R, Yang G, Liu D, et al. (2007) Validation and reproducibility of the food frequency questionnaire used in the Shanghai Men's Health Study. Br J Nutr 97, 993-1000.

25. Kleinbaum DG, Kupper LL \& Muller KE (1988) Variable reduction and factor analysis. In Applied Regression Analysis and Other Multivariable Methods, pp. 595-640. Boston, MA: PWS-Kent Publishing Company.

26. Hou X (2008) Urban-rural disparity of overweight, hypertension, undiagnosed hypertension, and untreated hypertension in China. Asia Pac J Public Health 20, 159-169.

27. Hermansen K (2000) Diet, blood pressure and hypertension. Br J Nutr 83, s113-s119.

28. Eberhardt MV, Lee CY \& Liu RH (2000) Antioxidant activity of fresh apples. Nature 405, 903-904.

29. McIntyre M, Bohr DF \& Dominiczak AF (1999) Endothelial function in hypertension: the role of superoxide anion. Hypertension 34, 539-545.

30. Czernichow S, Bertrais S, Blacher J, et al. (2005) Effect of supplementation with antioxidants upon long-term risk of hypertension in the SU.VI.MAX study: association with plasma antioxidant levels. J Hypertens 23, 2013-2018.

31. John JH, Ziebland S, Yudkin P, et al. (2002) Effects of fruit and vegetable consumption on plasma antioxidant concentrations and blood pressure: a randomised controlled trial. Lancet $\mathbf{3 5 9}$, 1969-1974.

32. Steffen LM, Kroenke CH, Yu S, et al. (2005) Associations of plant food, dairy product, and meat intake with $15-\mathrm{y}$ incidence of elevated blood pressure in young black and white adults: the Coronary Artery Risk Development in Young Adults (CARDIA) study. Am J Clin Nutr 82, 1169-1677.

33. Armstrong B, van Merwyk AJ \& Coates H (1997) Blood pressure in Seventh-day Adventist vegetarians. Am J Epidemiol 105, 444-449.

34. Chen Y, Factor-Lovak P, Howe GR, et al. (2006) Nutritional influence on risk of high blood pressure in Bangladesh: a populationbased cross-sectional study. Am J Clin Nutr 84, 1224-1232.

35. Ma G, Jin Y, Piao J, et al. (2005) Phytate, calcium, iron, zinc contents and their molar ratios in food commonly consumed in China. J Agric Food Chem 53, 10285-10290.

36. Lacour B, Tardivel S \& Drueke T (1997) Stimulation by citric acid of calcium and phosphorus bioavailability in rats fed a calcium-rich diet. Miner Electrolyte Metab 23, 79-87. 
37. Klatsky AL, Friedman GD, Siegelaub AB, et al. (1977) Alcohol consumption and blood pressure Kaiser-Permanente Multiphasic Health Examination data. $N$ Engl J Med 296, $1194-1200$.

38. Xin X, He J, Frontini MG, et al. (2001) Effects of alcohol reduction on blood pressure: a meta-analysis of randomized controlled trials. Hypertension 38, 1112-1117.

39. Marmot MG, Elliott P, Shipley MJ, et al. (1994) Alcohol and blood pressure: the INTERSALT study. BMJ 308, 1263-1267.

40. Yoshita K, Miura K, Morikawa Y, et al. (2005) Relationship of alcohol consumption to 7-year blood pressure change in Japanese men. J Hypertens 23, 1485-1490.
41. Tuomilehto J, Elo J \& Nissinen A (1982) Smoking among patients with malignant hypertension. Br Med J (Clin Res Ed) 284, 1086.

42. Primatesta P, Falaschetti E, Gupta S, et al. (2001) Association between smoking and blood pressure: evidence from the Health Survey for England. Hypertension 37, 187-193.

43. Okubo Y, Miyamoto T, Suwazono Y, et al. (2002) An association between smoking habits and blood pressure in normotensive Japanese men. J Hum Hypertens 16, 91-96.

44. Criqui MH, Langer RD \& Reed DM (1989) Dietary alcohol, calcium, and potassium; independent and combined effects on blood pressure. Circulation 80, 609-614.

\section{Appendix 1}

Factor loadings for three major dietary patterns at baseline for 39252 male adults in the Shanghai Men's Health Study*

\begin{tabular}{|c|c|c|c|c|c|c|}
\hline \multirow[b]{2}{*}{ No. } & \multicolumn{2}{|c|}{ Factor 1 (vegetable dietary pattern) } & \multicolumn{2}{|c|}{ Factor 2 (fruit and milk dietary pattern) } & \multicolumn{2}{|c|}{ Factor 3 (meat dietary pattern) } \\
\hline & Food items & Factor loading & Food items & Factor loading & Food items & Factor loading \\
\hline 1 & Green beans & 46 & Oranges & 55 & Chicken & 40 \\
\hline 2 & Yard long beans & 45 & Apples & 53 & Beef, lamb & 39 \\
\hline 3 & Cucumbers & 43 & Watermelon & 48 & Duck, goose & 38 \\
\hline 4 & Wax gourds & 42 & Pears & 47 & Organ meat & 38 \\
\hline 5 & Celery & 42 & Other fruits & 44 & Pig's feet & 37 \\
\hline 6 & Amaranth & 42 & Grapes & 43 & Eel & 34 \\
\hline 7 & Wild rice stems & 41 & Fresh milk & 42 & Shrimp, crab, etc. & 32 \\
\hline 8 & Tomatoes & 41 & Bananas & 41 & Ham hocks & 32 \\
\hline 9 & Chinese chives & 41 & Bread & 38 & Salt water fish & 31 \\
\hline 10 & Eggplant & 39 & Peaches & 38 & Noodles & 31 \\
\hline 11 & Potatoes & 39 & Desserts & 29 & Fresh pork (mixture) & 29 \\
\hline 12 & Asparagus lettuce & 38 & Preserved fruits & 23 & Pig liver & 28 \\
\hline 13 & Baby soyabeans & 38 & Edible tree fungi & 21 & Fried bean curd & 28 \\
\hline 14 & Garland chrysanthemums & 38 & Xianggu mushroom & 20 & Conch & 27 \\
\hline 15 & Clover & 38 & Eggs, duck eggs & 17 & Pork ribs & 26 \\
\hline 16 & Hyacinth beans & 37 & Milk powder & 9 & Fresh water fish & 20 \\
\hline 17 & Chinese cabbage & 36 & Soya milk & 8 & Fresh pork (lean) & 19 \\
\hline 18 & Cauliflower & 36 & Fresh pork (fat) & -14 & Pork chops & 15 \\
\hline 19 & Fresh peppers & 36 & & & Peanuts & 14 \\
\hline 20 & Garlic & 36 & & & Dried soyabeans & 12 \\
\hline 21 & Fresh mushrooms & 35 & & & Rice & -69 \\
\hline 22 & Bamboo shoots & 35 & & & & \\
\hline 23 & White turnips & 35 & & & & \\
\hline 24 & Luffa & 34 & & & & \\
\hline 25 & Spinach & 34 & & & & \\
\hline 26 & Green cabbage & 32 & & & & \\
\hline 27 & Onions & 32 & & & & \\
\hline 28 & Shepherd's purse & 32 & & & & \\
\hline 29 & Fresh peas & 31 & & & & \\
\hline 30 & Snow pea shoots & 31 & & & & \\
\hline 31 & Chinese greens (Bokchoi) & 30 & & & & \\
\hline 32 & Lotus root & 29 & & & & \\
\hline 33 & Soyabean sprouts & 24 & & & & \\
\hline 34 & Carrots & 24 & & & & \\
\hline 35 & Fresh broad beans & 24 & & & & \\
\hline 36 & Mung bean sprouts & 23 & & & & \\
\hline 37 & Heads of garlic & 23 & & & & \\
\hline 38 & Green onions & 23 & & & & \\
\hline 39 & Bean curd & 21 & & & & \\
\hline 40 & Mung beans, red beans & 21 & & & & \\
\hline 41 & Sea tangle & 21 & & & & \\
\hline 42 & Sea laver & 11 & & & & \\
\hline
\end{tabular}

${ }^{\star}$ Factor loadings are multiplied by 100 and rounded to the nearest integer. 